\title{
Effects of Weighted Despreading Sequence on CDMA Performance
}

\author{
Yuejin Huang and \\ yjhuang@eee.hku.hk \\ Tung Sang Ng \\ tsng@eee.hku.hk \\ Department of Electrical and Electronic Engineering, The University of Hong Kong, \\ Pokfulam Road, Hong Kong. Fax: +85225598738
}

\begin{abstract}
This paper addresses the effects of weighted despreading sequence on the performance of a coherent receiver in a DS-CDMA system. The chip weighting waveforms used in the receiver are designed for the purpose of multiple access interference rejection by emphasizing the transitions of the received signal of interest. Since the resulting weighted despreading sequence is determined only by one parameter, this leads to easy tuning of the weighted despreading sequence in practice to achieve the best performance. The criterion of tuning the parameter is to maximize the derived signal to interference plus noise ratio of the decision variable based on the relative strength between white noise and multiple access interference. As a result, according to the proposed approach, we can reject the multiple access interference without knowing co-user's spreading codes, timing, and phase since maximizing the signal to interference plus noise ratio in Gaussian noise minimizes the probability of error of the receiver.
\end{abstract}

\section{INTRODUCTION}

In a DS-CDMA system with perfect power control, a major limitation to the capacity is due to the multiple access interference (MAI). With the purpose of MAI rejection, the optimum multiuser receiver is studied in [1]-[2]. However, the resulting receiver is extremely complex. Based on the fact that the MAI can be modeled as zero-mean stationary colored Gaussion process, a simple receiver structure called integral equation receiver has been proposed in [3]. The integral equation receiver employs the despreading function which is the solution of a Fredholm integral equation of the second kind, and the resulting despreading function consists of $2 N^{2}$ exponential terms with $N(N+3)$ coefficients ( $N$ is processing gain). In practice, it is not easy to tune to the optimum despreading function when the processing gain $N$ is large. Based on the property that the despreading function given in [3] emphasizes the transitions in the received signal of the reference user for MAl rejection, we propose to weight the despreading sequence by exponential chip weighting waveforms[4]. Since the chip weighting waveforms employed is determined only by one parameter, this leads to easy tuning of the weighted despreding sequence in practice to achieve the best performance. In this paper, we evaluate various effects of the proposed despreading sequence on CDMA performance. Numerical results show that when the MAI is significant in a DS-CDMA system, the proposed receiver with adjustable despreading sequence performs far better than the conventional receiver with fixed despreading sequence. We also show that when the MAI is insignificant, change in the despreading function has little effect on system performance.

\section{SYSTEM MODEL}

Suppose there are $K$ CDMA users accessing the channel. User $k$ transmits a data sequence $b_{k}(t)$ and employs a spreading sequence $a_{k}(t)$ to spread each data bit. The spreading and data sequences for the $k$ th user are given by $a_{k}(t)=\sum_{j m-\infty}^{\infty} a_{j}^{(k)} P_{T_{i}}\left(t-j T_{c}\right) \quad$ and $b_{k}(t)=\sum_{j=-\infty}^{\infty} b_{j}^{(k)} P_{x_{i}}\left(t-j T_{b}\right)$ respectively where $P_{x}(y)=1$ for $0<\mathrm{y}<\mathrm{x}$ and 0 otherwise. In our study, $b_{j}^{(k)}$ and $a_{j}^{(k)}$ are modeled as independent random variables taking values -1 or +1 with equal probabilities. It is assumed that there are $N$ chips of a spreading sequence in the interval of each data bit $T_{b}$ and the spreading sequence has period equal to $N$. In other words, $T_{b}=N T_{c}$ and $a_{j}^{(k)}=a_{j+N}^{(k)}$ for $-\infty<j<+\infty$. The transmitted signal for the $k$ th user is

$$
S_{k}(t)=\sqrt{2 P} b_{k}(t) a_{k}(t) \cos \left(\omega_{0} t+\theta_{k}\right)
$$

where the transmitted power $P$ and the carrier frequency $\omega_{0}$ are common to all users, and $\theta_{k}$ is the phase angle of the $k$ th transmitter. For an asynchronous DS-CDMA system the received signal at the base station can be represented as

$$
\begin{aligned}
r(t) & =\sum_{k=1}^{K} S_{k}\left(t-\tau_{k}\right)+n(t) \\
& =\sqrt{2 P} \sum_{k=1}^{K} b_{k}\left(t-\tau_{k}\right) a_{k}\left(t-\tau_{k}\right) \cos \left(\omega_{0} t+\phi_{k}\right)+n(t)
\end{aligned}
$$


where $K$ denotes the number of active users, $\tau_{k}$ and $\phi_{k}\left(=\theta_{k}-\omega_{0} \tau_{k}\right)$ for $1 \leq k \leq K$ are random time delays and phases along the communications links between the $K$ transmitters and the particular receiver, respectively, and $n(t)$ is additive white Gaussian noise (AWGN) with two-side spectral density $N_{0} / 2$. The random variables $\left\{\tau_{k}\right\}$ and $\left\{\phi_{k}\right\}$ are independent of one another and uniformly distributed in $\left[0, T_{h}\right]$ and $[0,2 \pi]$, respectively.

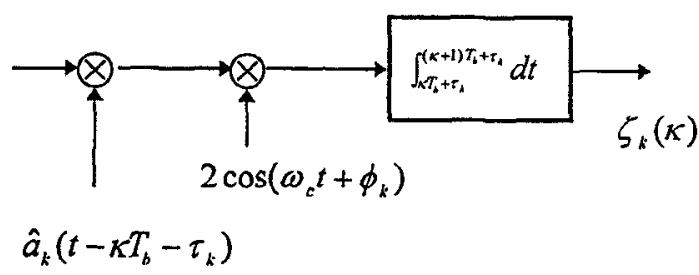

Fig. 1 The structure of a coherent receiver with weighted despreading sequence.

For BPSK modulation, the structure of the proposed coherent receiver with weighted despreading sequence for the $k$ th user is shown in Fig. 1 where $\zeta_{k}(x)$ is the decision variable and $\hat{a}_{k}(t)$ is the weighted despreading sequence which will be stated in detail below.

\section{SYSTEM PERFORMANCE}

\section{A. The Weighted Despreading Sequences}

In a DS - CDMA system, the MAI is the sum of many independent co-user signals so that it can be modeled as zero-mean stationary colored Gausssian process. When MAI and AWGN occur simutaniously, the optimum CDMA receiver in this case for MAI rejection is called integral equation receiver [3] in which the despreading function $q(t)$ for the $k$ th receiver should be the solution to the integral equation

$$
\int_{0}^{T_{b}} C_{n}(t-\tau) q(t) d \tau=-N_{0} q(t)+a_{k}(t), 0 \leq t \leq T_{b}
$$

where $C_{n}(\tau)$ is the autocorrelation function of the MAI. Eq.(3) is known as Fredholm integral equation of the second kind. The integral equation (3) has been solved in [3] for the case in which the spreading pulses are rectangular and resulting despreading function $q(t)$ consists of $2 N^{2}$ exponential terms with $N(N+3)$ coefficients. However it is not easy to tune to the optimum despreading function $q(t)$ for MAI rejection when processing gain $N$ is large. To overcome the tuning problem, we proposed a weighted despreading sequence for the $k$ th receiver $[4,5]$ as follows

$$
\hat{a}_{k}(t)=\sum_{j=1}^{\infty} a_{j}^{(k)} w_{j}^{(k)}\left(t-j T_{c} \mid\left\{c_{j}^{(k)}, c_{j+1}^{(k)}\right\}\right) P_{r}\left(t-j T_{c}\right)
$$

where $c_{j}^{(k)}=a_{j-1}^{(k)} a_{j}^{(k)}$ and $w_{j}^{(k)}\left(t \mid\left\{c_{j}^{(k)}, c_{j+1}^{(k)}\right\}\right)$ for $0 \leq t \leq T_{c}$ is the $j$ th chip weighting waveform conditioned on the status of three consecutive chips $\left\{a_{j-1}^{(k)}, a_{j}^{(k)}, a_{j+1}^{(k)}\right\}$. In our analysis, we choose the $j$ th chip weighting waveforms as

$$
w_{j}^{(k)}\left(t \mid\left\{c_{j}^{(k)}, c_{j+1}^{(k)}\right\}\right)= \begin{cases}c w_{1}(t) & \text { if } c_{j}^{(k)}=+1 \text { and } c_{j+1}^{(k)}=+1 \\ c w_{2}(t) & \text { if } c_{j}^{(k)}=-1 \text { and } c_{j+1}^{(k)}=-1 \\ c w_{3}(t) & \text { if } c_{j}^{(k)}=-1 \text { and } c_{j+1}^{(k)}=+1 \\ c w_{4}(t) & \text { if } c_{j}^{(k)}=+1 \text { and } c_{j+1}^{(k)}=-1\end{cases}
$$

where $c w_{p}(t)$ for $p \in[1,2,3,4]$ are the chip weighting waveforms which will be described in detail below. Based on the property that the optimum despreading function given in [3] emphasizes the transitions of the received signal of interest user, we therefore define the elements of the chip weighting waveform vector $\left\{c w_{1}(t), c w_{2}(t), c w_{3}(t), c w_{4}(t)\right\}$ as follows

$$
\begin{aligned}
& c w_{\mathrm{l}}(t)=e^{-\gamma / 2} P_{T_{\mathrm{t}}}(t) \\
& c w_{2}(t)=e^{-\gamma t / \tau_{0}} P_{T / 2}(t)+e^{-\gamma\left(1-t / T_{c}\right)} P_{T_{\ell} / 2}\left(t-T_{c} / 2\right) \\
& c w_{3}(t)=e^{-y t / \tau_{c}} P_{t_{c} / 2}(t)+e^{-\gamma / 2} P_{r_{c} / 2}\left(t-T_{c} / 2\right) \\
& c W_{4}(t)=e^{-\gamma / 2} P_{T, 2}(t)+e^{-\gamma\left(1-t / T_{c}\right)} P_{T, 12}\left(t-T_{c} / 2\right)
\end{aligned}
$$

where $\gamma \in[0, \infty)$ is a parameter of the chip weighting waveforms. When $\gamma=0$ in ( 6 ), the chip weighting waveforms $c w_{p}(t)$ for $p \in[1,2,3,4]$ reduce to the same rectangular pulse $P_{T_{e}}(t)$. Fig. 2 shows the diagrams of the chip weighting waveforms.

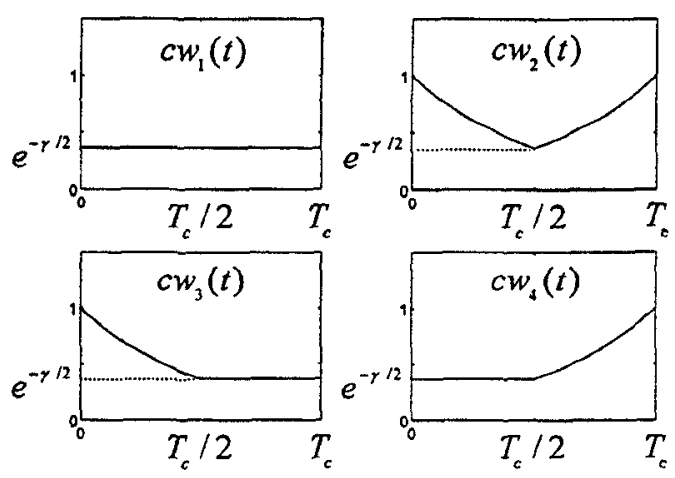

Fig. 2 The exponential chip weighting waveforms.

\section{B. Performance Analysis}

We arbitrarily choose the $i$ th user as the reference user and analyze the performance of the proposed coherent receiver for data symbol $b_{i}^{(i)}$. After demodulation, the conditional output random variable of the reference user's receiver, denoted by $\zeta_{i}(\lambda)$, can be expressed as 


$$
\begin{array}{r}
\zeta_{i}(\lambda)=\int_{\lambda T_{i}+\varepsilon_{i}}^{\langle\lambda+1\rangle T_{b}+\tau_{i}}\left\{2 r(t) \hat{a}_{i}\left(t-\lambda T_{b}-\tau_{i}\right)\right. \\
\left.\cdot \cos \left(\omega_{0} t+\phi_{i}\right)\right\} d t
\end{array}
$$

Since the carrier frequency $f_{0}$ is much large than $T_{b}^{-1}$ in practical system, the double-frequency terms in ( 7 ) can be ignored and Eq. ( 7 ) reduces to

$$
\zeta_{i}(\lambda)=S_{i}\left(\lambda \mid\left\{c_{j}^{(i)}\right\}\right)+N_{i}\left(\lambda \mid\left\{c_{j}^{(i)}\right\}\right)+\sum_{k=1,(k+i)}^{K} Y_{\left\langle c_{j}^{(i)}\right\}}^{(k j)}
$$

where the first, second, and third components are the conditional desired, noise, and multiple access interference components. The desired signal term of the $S_{i}\left(\lambda \mid\left\{c_{j}^{(i)}\right\}\right)$ in $(8)$, conditioned on $\left\{c_{j}^{(i)}\right\}$, is given by [5]

$$
\begin{aligned}
& S_{i}\left(\lambda \mid\left\{c_{j}^{(i)}\right\}\right) \\
& \quad=b_{i}^{(i)} \sqrt{2 P}\left[2 \hat{N}_{i} T_{c}\left(1-e^{-y / 2}\right) / \gamma+\left(N-\hat{N}_{i}\right) T_{c} e^{-\gamma}\right]
\end{aligned}
$$

where $\hat{N}_{i}$ is a random variable which represents the number of times of occurrence that $c_{j}^{(i)}=-1$ for all $j$ $\in[0, N-1]$. The variance of the white noise term $N_{i}\left(\lambda \mid\left\{c_{j}^{(i)}\right\}\right)$ in (8), conditioned on $\left\{c_{j}^{(i)}\right\}$ and denoted by $\sigma_{N\left(c_{c}^{(j)}\right)}^{2}$, is given by [5]

$$
\sigma_{\left.N c_{c}^{(\prime \prime)}\right)}^{2}=N_{0}\left[\hat{N}_{i} T_{c}\left(1-e^{-\gamma / 2}\right) / \gamma+\left(N-\hat{N}_{i}\right) T_{c} e^{-\gamma / 2}\right]
$$

The third term in ( 8 ) is the multiple access interference term of the reference receiver with the conditional variance, conditioned on $\left\{c_{j}^{(i)}\right\}$, given by [5]

$$
\sigma_{I K c_{j}^{(j)\}}}^{2}=\frac{N T_{c}^{2}(K-1) P}{\gamma^{2}} e^{-\gamma} \Xi^{(e)}\left(\Gamma^{\left(c_{j}^{(j)}\right\}}, \gamma\right)
$$

where

$$
\begin{aligned}
& \Xi^{(e)}\left(\Gamma^{\left(c^{(i)}\right)}, \gamma\right)=\frac{1}{N}\left\{\Gamma_{\{-1,-1,-1)}^{(i)}\left[4+\frac{12}{\gamma}-\frac{16}{\gamma} e^{\gamma / 2}+\frac{4}{\gamma} e^{\gamma}\right]\right. \\
& +\left(\Gamma_{\{-1,-1,1\}}^{(i)}+\Gamma_{\{1,-1,-1\}}^{(i)}\right)\left[\frac{5}{2}-\frac{\gamma}{4}+\frac{\gamma^{2}}{24}+\frac{19}{2 \gamma}+e^{\gamma / 2}-\frac{12 e^{\gamma / 2}}{\gamma}+\frac{5 e^{\gamma}}{2 \gamma}\right] \\
& +\left(\Gamma_{\{-1,1,1\}}^{(i)}+\Gamma_{\{1,1,-1\}}^{(i)}\right)\left[-\frac{3}{2}-\frac{3}{4} \gamma+\frac{19}{24} \gamma^{2}-\frac{1}{2 \gamma}+e^{\gamma / 2}+\frac{e^{\gamma}}{2 \gamma}\right] \\
& +\Gamma_{\{-1,1,-1\}}^{(i)}\left[-3-\frac{3}{2} \gamma+\frac{7}{12} \gamma^{2}-\frac{1}{\gamma}+2 e^{\gamma / 2}+\frac{e^{\gamma}}{\gamma}\right] \\
& \left.+\Gamma_{\{(1,-1,1\}}^{(i)}\left[1-\frac{\gamma}{2}+\frac{\gamma^{2}}{12}+\frac{7}{\gamma}+2 e^{\gamma / 2}-\frac{8}{\gamma} e^{\gamma / 2}+\frac{e^{\gamma}}{\gamma}\right]+\gamma^{2} \Gamma_{\{1,1,1\}}^{(i)}\right]
\end{aligned}
$$

and $\Gamma_{\left\{v_{1}, v_{2}, v_{j}\right\}}^{\langle i\rangle}$ is the number of times of occurrence that $\left\{c_{j-1}^{(i)}, c_{j}^{(i)}, c_{j+1}^{(i)}\right\}=\left\{v_{1}, v_{2}, v_{3}\right\}$ for all $j \in[0, N-1]$ in the reference user's spreading sequence and each $v_{n}$, $n \in[1,2,3]$, is a random variable of elements of $\{+1,-1\}$. It is clear that $\sum_{\left\{v_{1}, v_{z}, v_{1}\right\}} \Gamma_{\left\{v_{1}, v_{2}, v_{\}}\right\}}^{\langle i}=N$.

By definition, the conditional signal to interference plus noise ratio of the decision variable is expressed as

$$
\operatorname{SINR} R_{i}=\left\{\frac{\sigma_{\left.N \mid c_{j}^{(i)}\right\}}^{2}}{\left[S\left(\lambda \mid\left\{c_{j}^{(i)}\right\}\right)\right]^{2}}+\frac{\sigma_{\left\langle\left\{c_{j}^{(i)}\right\}\right.}^{2}}{\left[S\left(\lambda \mid\left\{c_{j}^{(i)}\right\}\right)\right]^{2}}\right\}^{-1}
$$

where $S\left(\lambda \mid\left\{c_{j}^{(i)}\right\}\right), \sigma_{\left.N \nVdash c_{j}^{(i)}\right\}}^{2}$ and $\sigma_{I\left\{c_{f}^{(i)}\right\}}^{2}$ are expressed by (9), (10) and (11), respectively. Substituting (9), (10) and ( 11 ) into ( 13$)$, the conditional signal to interference plus noise ratio, conditioned on $\left\{c_{j}^{(i)}\right\}$, is given by

$$
\begin{gathered}
\operatorname{SINR}_{t}=\left\{\frac{\gamma\left[\chi\left(1-e^{-\gamma}\right)+\gamma(1-\chi) e^{-\gamma}\right]}{2 \bar{\gamma}_{b}\left[2 \chi\left(1-e^{-\gamma / 2}\right)+\gamma(1-\chi) e^{-\gamma / 2}\right]^{2}}\right. \\
\left.+\frac{(K-1) \Xi^{(e)}\left(\Gamma^{\left(c^{(/)}\right)}, \gamma\right)}{2 N\left[2 \chi\left(e^{\gamma / 2}-1\right)+\gamma(1-\chi)\right]^{2}}\right\}^{-1}
\end{gathered}
$$

where $E_{b}=P T_{b}, \bar{\gamma}_{b}=E_{b} / N_{0}$ and $\chi=\hat{N}_{i} / N$.

\section{NUMERICAL RESULTS}

In this section, we present numerical results on the performance of the proposed coherent receiver. In all the bit error rate ( BER ) curves, the BER is defined as $B E R=Q(\sqrt{\operatorname{SINR}})$ where

$$
Q(x)=\left(\frac{1}{2 \pi}\right) \cdot \int_{x}^{\infty} e^{-t^{2} / 2} d t
$$

In the following, it is assumed that, in the reference user's random spreading sequence, $\quad N=127, \quad \hat{N}_{i}=62$, $\Gamma_{\{-1,-1,-1\}}^{(i)}=10, \Gamma_{\{-1,-1,1)}^{(i)}+\Gamma_{\{1,-1,-1\}}^{(i)}=36, \Gamma_{\{-1,1,1\}}^{(i)}+\Gamma_{\{1,1,-1\}}^{(i)}=32$, $\Gamma_{\{-1,1,-1\}}^{(i)}=18, \Gamma_{\{1,-1,1\}}^{(i)}=16$, and $\Gamma_{\{1,1,1\}}^{(i)}=15$.

In Fig. 3, SINR, is plotted using the parameters $\gamma$ as the independent variables for two values of the signal to white noise ratios $\bar{\gamma}_{b}$ when $K=37$. It is clear that the weighted despreading sequence reduces to rectangular spreading sequence when $\gamma=0$. Therefore, $S I N R_{i}$ at $\gamma=0$ takes the value equal to the $S I N R_{i}$ derived in the case that the chip waveforms of the reference despreading sequence are rectangular. Fig. 3 shows that the parameter 
$\gamma$ should be tuned with respect to each $\bar{\gamma}_{b}$ in order to maximize the SINR when $\bar{\gamma}_{h}$ is relatively large. From this figure one can see that the maximum value of SINR

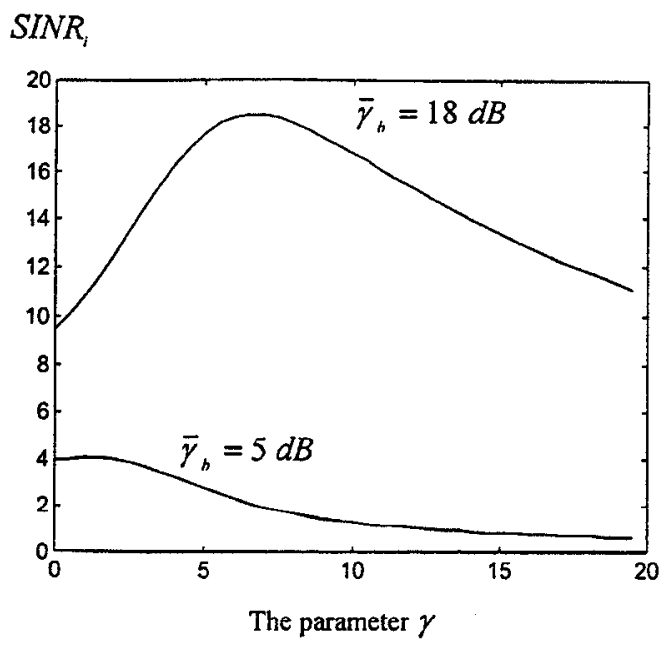

Fig. 3 SINR versus the parameter $\gamma$ for two values $\bar{\gamma}_{b}$ when $N=127$ and $K=37$.

obtained by tuning the parameter $\gamma$ is very close to the value of SINR at $\gamma=0$ when the MAI is insignificant. For example, when $\bar{\gamma}_{b} \leq 5 d B$, the maximum value of SINR, obtained by tuning the parameter $\gamma$ can simply be replaced by the value of $S I N R_{i}$ at $\gamma=0$ with a negligible loss of SINR. In other words, the change to the despreading function has little effect on system SINR, when the AWGN is significant. This is shown in Fig. 4.

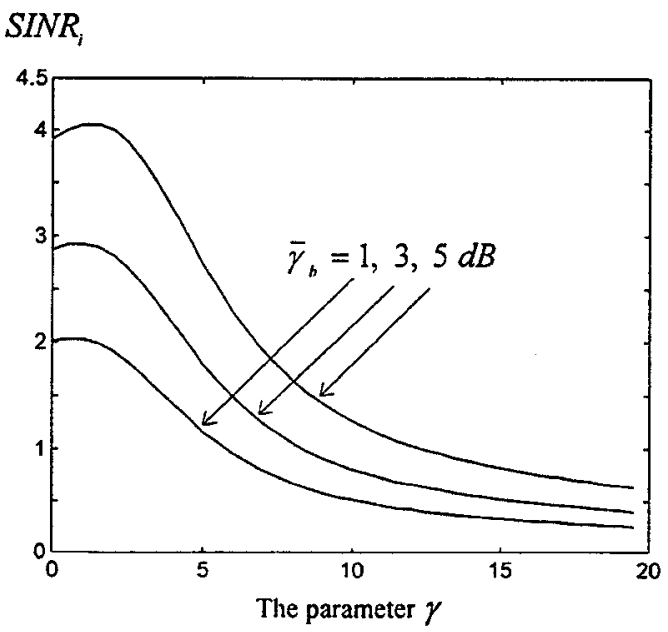

Fig. 4 SINR, versus the parameter $\gamma$ at $N=127$ and $K=37$ when $\bar{\gamma}_{b} \leq 5 d B$

Fig. 5 shows the optimum values of the parameter $\gamma_{\text {apt }}$ which maximizes the SINR versus the number of active users $K$. It is clear that the optimum value of the parameter $\gamma_{o p t}$ increases as the signal to noise ratio $\bar{\gamma}_{b}$ increases.

Fig.6 shows the BER performance of the proposed coherent receiver for the cases of $\gamma=0$ and $\gamma$ tuned to maximize the SINR . The dashed line is the BER for the

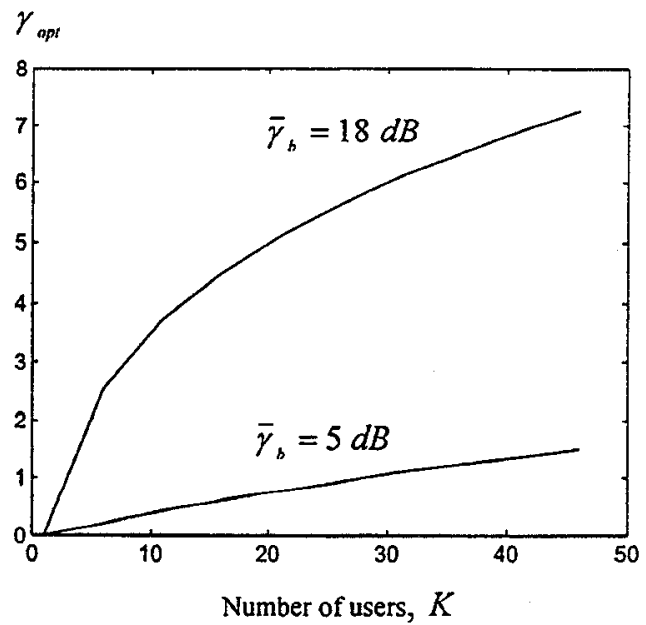

Fig. 5 The parameter $\gamma_{\text {opt }}$ versus the number of users $K$ for two values of $\bar{\gamma}_{b}$ when $N=127$

receiver when the despreading sequence is the rectangular spreading sequence $(\gamma=0)$. The solid line corresponds to the BER for the receiver in which the parameters $\gamma$ of the weighted despreading sequence is tuned explicitely to each $\bar{\gamma}_{b}$ to maximize the SINR. It is clear that the performance of the receiver with the weighted despreading sequences over the conventional receiver with the rectangular despreading sequences is dramatic, although the weighted despreading sequences are not exactly the solution of the integral equation.

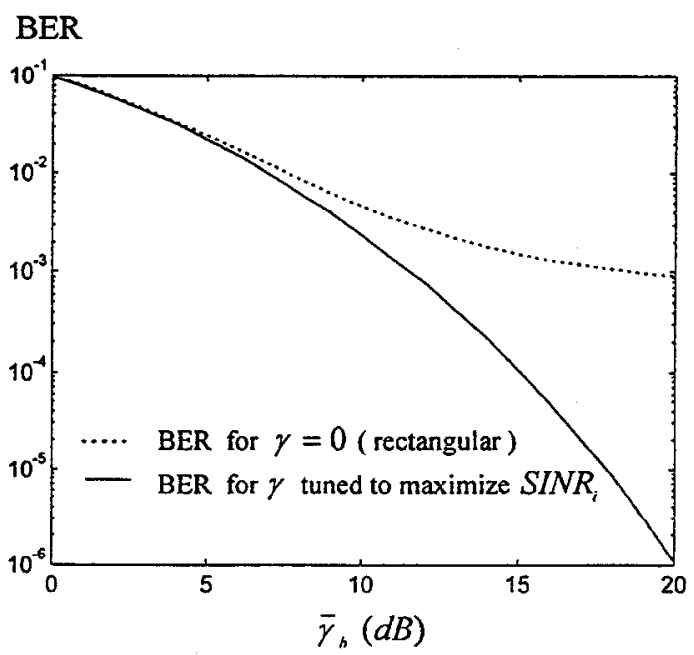

Fig. 6 BER versus $\bar{\gamma}_{b}$ for both rectangular and adjustable despreading function when $N=127$ and $K=37$. 


\section{CONCLUSIONS}

In this paper, we have introduced a coherent CDMA receiver in which the despreading sequence is weighted by adjustable chip waveforms. The chip weighting waveforms are designed for the objective of multiple access interference rejection based on the property of the optimum despreading function given in [3] that the despreading sequence emphasizes the transitions in the received spreading sequence of interest. To achieve the maximum value of the system $\operatorname{SINR}_{i}$, we can simply tune the parameter $\gamma$ of the weighted despreading sequence based on the relative strenght between MAI and AWGN. When the MAI is significant, the receivers using the despreading sequence weighted by adjustable chip waveforms outperforms greatly the conventional receiver using the despreading sequence with fixed or rectangular chip pulses, without increasing a lot in complexity.

\section{ACKNOWLEDGMENT}

This work was supported by the Research Grants Council of Hong Kong and the CRCG of The University of Hong Kong.

\section{REFERENCES}

[1] S. Verdú, " Minimum probability of error for asynchronous Gaussian multiple-access channels," IEEE Tran. Inform. Theory, vol. IT-32, pp. 85-96, Jan. 1986.

[2] Z. Xie, R. T. Short, and C. K. Rushforth, "A family of suboptimum detectors for coherent multiuser communications," IEEE J. Select. Areas Commun., vol. 8, pp. 683-690, May 1990.

[3] A. M. Monk, M. Davis, L. B. Milstein, and C. H. Helstrom, "A Noise-Whitening Approach to Multiple Access Noise Rejection - Part I: Theory and Background," IEEE J. Select. Areas Commun., vol. 12, No. 5, pp. 817-827, June 1994.

[4] Y. Huang and T. S. Ng, "Performance of Coherent Receiver with Weighted Despreading Sequence for DS-CDMA," Electron. Lett., vol. 33, pp. 23-25, Jan. 1997

[5] Y. Huang and T. S. Ng, "A DS-CDMA System Using Despreading Sequences Weighted by Adjustable Chip Waveforms," submitted for publication. 